\title{
Evaluation of the Pulmonary Vasculature and Dynamics with Intravascular Ultrasound Imaging in Children and Infants
}

\author{
R. M. F. BERGER, A. H. CROMME-DIJKHUIS, A. M. VAN VLIET, AND J. HESS \\ Department of Pediatrics, Division of Pediatric Cardiology, Sophia Children's Hospital/University \\ Hospital Rotterdam, Rotterdam, The Netherlands
}

\section{ABSTRACT}

\begin{abstract}
The ability to assess the pulmonary vasculature in pulmonary vascular disease by hemodynamic or histologic evaluation is limited. We sought to determine the feasibility of intravascular ultrasound techniques in infants and children and to assess simultaneously the morphology and dynamics of pulmonary arteries. Patients were seen in the Department of Pediatrics, Division of Pediatric Cardiology, Sophia Children's Hospital, Rotterdam, The Netherlands. We performed intravascular ultrasound imaging in 11 pediatric patients with congenital heart disease undergoing cardiac catheterization. Luminal diameter, area, and pulsatility were determined at two to five sites in the pulmonary branches. Pulmonary vascular reaction to $100 \%$ oxygen inhalation was studied. Patients weighed $4.1-51.0 \mathrm{~kg}(21.8$ $\pm 16.3 \mathrm{~kg}$, mean $\pm \mathrm{SD}$ ). Luminal diameters, areas, and pulsatilities could be determined reproducibly in arteries with diameters from 1.6 to $9.3 \mathrm{~mm}$. In total 39 sites were studied in 11 patients. Pulsatility was related to vessel size $(r=0.81)$, although a substantial interindividual variation was present. After $100 \%$ oxygen inhalation, pulsatility increased in all arteries
\end{abstract}

(from $20.0 \pm 3.3 \%$ to $25.9 \pm 2.9 \%, p<0.05$ ), and vasodilatation could be directly visualized, most prominently in the smallest arteries (percentage change in diameter, mean $7.4 \pm 2.8 \%$ versus $-2.8 \pm 3.1 \%$ in the largest arteries, $p<0.001$ ). Measurement of wall thickness was not feasible, but specific changes in the appearance of the wall structure could be recognized in a patient with severely elevated pulmonary vascular resistance. The specific advantages of intravascular ultrasound in assessing pulmonary vascular disease are discussed. We conclude that intravascular ultrasound imaging of the pulmonary vasculature is feasible in infants and children and provides a unique opportunity to assess directly pulmonary dynamics in vivo. Therefore, it may be a valuable tool in evaluating the pulmonary vasculature and its responses to normal and pathologic conditions. (Pediatr Res 38: $36-41,1995$ )

Abbreviations
PVD, pulmonary vascular disease
IVUS, intravascular ultrasound

The state of the pulmonary vasculature plays a crucial role in the management and prognosis of children with congenital heart disease $(1,2)$. To assess the pulmonary vasculature, mainly two diagnostic tools are currently in use: hemodynamic evaluation by cardiac catheterization and examination of lung histology. Although both techniques provide valuable information, both also have important limitations. One must realize that, on the one hand, hemodynamic data, such as pulmonary artery pressure, blood flow, and resistance, reflect merely indirect consequences of the vascular process; moreover, they are based on various assumptions and, thus, inevitably are accompanied by potential errors (2). On the other hand, histologic examination of lung biopsy tissue provides a qualitative and quantitative description of morphologic changes in the vascular wall, but remains a static, in vitro examination without

Received December 8, 1994; accepted February 21, 1995.

Correspondence and reprint requests: R. M. F. Berger, M.D., Sophia Children's Hospital, Dr. Molenwaterplein 60, 3015 GJ Rotterdam, The Netherlands.

Manuscript dedicated to Professor H. K. A. Visser in honor of his retirement. functional validation $(3,4)$. Furthermore, it requires a thoracotomy. These limitations are illustrated by reports of the correlation of the two techniques, showing important discrepancies in individual patients with PVD (5-8).

It has been shown that by means of IVUS imaging vascular luminal diameter, area, and pulsatility can be determined accurately, as well as qualitative and quantitative aspects of the vascular wall $(9-12)$. We hypothesized that with this technique it is possible to assess directly and simultaneously vessel wall appearance and "real time" vessel wall dynamics in vivo in peripheral pulmonary arteries of children with congenital heart disease. This would give direct, unique information on the appearance and functional state of the pulmonary vascular bed, which is directly available during cardiac catheterization and might obviate the need for a thoracotomy. We therefore performed a study to determine the feasibility of IVUS techniques in infants and children and to assess the appearance and the dynamics of the pulmonary vasculature in vivo. 


\section{METHODS}

Eleven patients, requiring diagnostical or therapeutical cardiac catheterization because of congenital heart disease in the Sophia Children's Hospital, Rotterdam, the Netherlands, underwent simultaneous IVUS imaging of the pulmonary arteries. IVUS was performed using disposable ultrasound catheters, 4.2F CVIS (Cardiovascular Imaging Systems Inc., Sunnyvale, CA) or $3.5 \mathrm{~F}$ Sonicath (Boston Scientific Corp., Watertown, MA), both having a $30-\mathrm{MHz}$ transducer at its tip. These were connected to the CVIS Insight system or the HP Sonos Intravascular Imaging System (Hewlett Packard, Andover, MA), respectively. All patients underwent complete right heart catheterization, under general anesthesia, including determination of pulmonary artery pressure, capillary wedge pressure, pulmonary blood flow calculated by the Fick principle, and pulmonary vascular resistance. A $6 \mathrm{~F}, 70-\mathrm{cm}$ long sheath (Cordis, Miami, FL) was advanced from the femoral vein into the proximal pulmonary artery. Through this sheath the ultrasound catheter was directed along the pulmonary branch, toward a wedged position. At consecutive sites, from the proximal artery, via the segment arteries to the peripheral arteries, identified and defined by fluoroscopy (13), ultrasound images were recorded, simultaneously with the ECG, and analyzed off-line. Pulmonary artery diameter and area were measured at end-diastolic (minimal) and peak-systolic (maximal) dimensions. Vascular pulsatility was defined as the difference between planimetered peak-systolic and end-diastolic areas divided by end-diastolic area $\times 100 \%$. The average value of three cardiac cycles was used for analysis. In six of 11 patients all measurements were repeated at identical sites after inhalation of $100 \%$ oxygen as a pulmonary vasodilator. Written informed consent was obtained from the parents of all children. The study protocol was approved by the Medical Ethical Committee of the University Hospital Rotterdam.

Statistical analysis. Data are expressed as mean values and standard deviations. Correlations were calculated using linear regression and correlation coefficient. $t$ test and Wilcoxon rank sum test were used to evaluate the effect of oxygen inhalation. A $p$ value $<0.05$ was considered significant.

\section{RESULTS}

IVUS imaging of the pulmonary arteries was performed in 11 children. Patients characteristics are shown in Table 1. Pulmonary vascular resistance was normal in eight patients, slightly to moderately elevated in two, and severely elevated in one patient. In all patients the positioning of the long sheath in the proximal pulmonary artery and guiding of the ultrasound catheter through this sheath along the pulmonary branch could be achieved easily. Once the catheter was placed in a stable, nonbranching position, the obtained images were of good quality in all patients (Fig. 1). There was a clear delineation between blood and the inner vessel wall, allowing reproducible measurements of systolic and diastolic vessel dimensions in all recordings. Measurements sites are shown in Figure 2. Enddiastolic diameter of the vessels reached varied from 1.6 to 9.3 $\mathrm{mm}$. Larger arteries could not be visualized adequately because of limited penetration power of the $30-\mathrm{MHz}$ transducer. The pulsatility of the pulmonary artery varied at different sites in the arterial branch and showed a relation with the size of the vessel, expressed as end-diastolic area. In all patients but one (patient 11) the pulsatility increased in the more proximal vessels. The relation for the total patient group $(r=0.81 ; p<$ 0.05 ) is shown in Fig. $3 A$. Between the individual patients there was a substantial variation in pulsatility per vessel diameter and in the extent of pulsatility increase (Fig. $3 B$ ). In six patients the reaction on $100 \%$ oxygen inhalation was studied. Pulsatility increased in all vessels. The larger vessels tended to show the largest increase in pulsatility (Fig. 4). Only patient 11 showed a different reaction pattern. The mean pulsatility in this patient was smaller than the mean pulsatility in the other patients $(9.5 \pm 0.7 \%, n=2$ versus $21.5 \pm 13.6 \%, n=14)$. Furthermore, the increase in pulsatility after oxygen inhalation tended to be diminished $(13.5 \pm 0.7 \%$ versus $27.6 \pm 13.5 \%)$. End-diastolic diameters increased after oxygen inhalation. This reaction was most prominent in the smallest vessels (percentage change in diameter, $4-11 \%$; mean, $7.4 \pm 2.8 \% ; p=0.001$ ) and was less prominent in the medium sized vessels $(2-6 \%$; mean, $4.2 \pm 1.8 \% ; p=0.002$ ). There was a slight decrease in diameter in the largest vessels ( -6 to $-2 \%$; mean, $-2.8 \pm$

Table 1. Patient characteristics

\begin{tabular}{|c|c|c|c|c|c|c|c|}
\hline Patient & Age (y) & $\begin{array}{l}\text { Weight } \\
(\mathrm{kg})\end{array}$ & Diagnosis & $\begin{array}{c}\text { mPAP } \\
(\mathrm{mm} \mathrm{Hg})\end{array}$ & $\begin{array}{c}\mathrm{Qp} \\
\left(\mathrm{L} / \mathrm{min} / \mathrm{m}^{2}\right) \\
\end{array}$ & $\begin{array}{l}\text { PVR 30\% } \\
\left(\text { WU.m }{ }^{2}\right)\end{array}$ & $\begin{array}{c}\text { PVR } 100 \% \\
\left(\text { WU. } \mathrm{m}^{2}\right)\end{array}$ \\
\hline 1 & 3.1 & 15.6 & PDA & 12 & 4.3 & 1.0 & 0.2 \\
\hline 2 & 11.4 & 51.0 & PDA & 17 & 6.0 & 1.2 & - $^{*}$ \\
\hline 3 & 3.3 & 15.4 & PDA & 11 & 6.7 & 0.2 & - \\
\hline 4 & 3.0 & 15.8 & PDA & 11 & 5.3 & 0.8 & - \\
\hline 5 & 6.5 & 24.7 & PDA & 13 & 4.4 & 0.9 & - \\
\hline 6 & 0.7 & 5.3 & VSD & 24 & 8.6 & 1.6 & 1.7 \\
\hline 7 & 11.5 & 48.0 & ASDII & 13 & 4.4 & 1.6 & - \\
\hline 8 & 5.0 & 12.0 & ASDII & 12 & 7.3 & 1.0 & - \\
\hline 9 & 16.0 & 36.5 & ASDI & 24 & 4.3 & 4.2 & 2.3 \\
\hline 10 & 0.4 & 4.1 & AVSD & 33 & 4.2 & 5.5 & 2.8 \\
\hline 11 & 4.4 & 11.7 & AVSD & 72 & 4.4 & 15.0 & 19.0 \\
\hline Mean & 6.0 & 21.8 & & 22 & 5.5 & 3.0 & \\
\hline (SD) & 5.1 & 16.3 & & 18 & 1.5 & 4.3 & \\
\hline
\end{tabular}

ASDII, atrial septal defect of the secundum type; AVSD, atrioventricular septal defect; PDA, patent ductus arteriosus; VSD, ventricular septal defect; mPAP, mean pulmonary artery pressure; Qp, pulmonary blood flow; PVR 30\%, pulmonary vascular resistance with 30\% oxygen inhalation; PVR 100\%, idem with $100 \%$ oxygen inhalation. WU, Wood's units.

* IVUS study, but no hemodynamic data during $100 \%$ oxygen inhalation. 


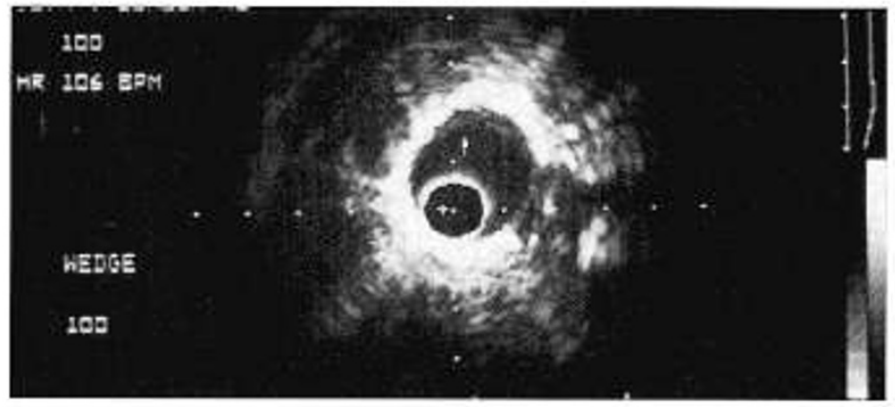

Figure 1. IVUS image of a peripheral pulmonary artery of patient $11 .+$, Ultrasound catheter. Note the gray layer at the inner border of the vessel wall (arrow). Distance between two white points $(O)$ is $1 \mathrm{~mm}$.

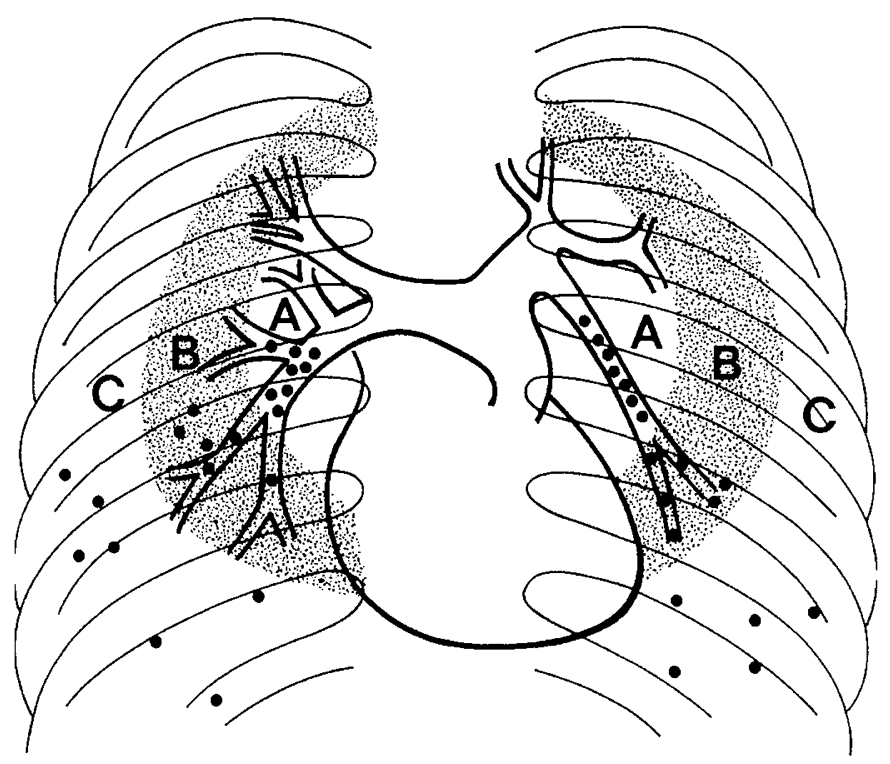

Figure 2. Measurement sites in the pulmonary vascular tree. $A$, Proximal arteries, luminal diameter, 4.2-9.3 mm (6.4 $\pm 1.3 \mathrm{~mm}$, mean $\pm \mathrm{SD}), n=15$; $B$, Segment arteries, luminal diameters, $2.3-5.9 \mathrm{~mm}(3.9 \pm 1.1 \mathrm{~mm}), n=12$; $C$, Peripheral arteries, luminal diameters. $1.6-2.9 \mathrm{~mm}(2.3 \pm 0.4 \mathrm{~mm}), n=12$. $n=$ number of measurement sites.

3.1\%; $p=0.047$ ) (Fig. 5). Again, this reaction pattern was similar in all patients except patient 11 . In this patient the end-diastolic diameter of the largest vessels increased $2 \%$.

The adventitial border could not be delineated adequately (Fig. 1). Consequently, wall thickness could not be measured. However, there was a specific appearance of the wall aspect in patient 11 , in whom a gray layer could be distinguished at the inner border of the vessel wall. Such a layer was not seen in the other patients. There were no procedure-related complications in any of the patients.

\section{DISCUSSION}

Our data show that IVUS imaging of the pulmonary arteries in infants and children is feasible. There was a clear delineation between blood and the inner vessel wall, allowing reproducible measurements of systolic and diastolic vessel dimensions. Accuracy of vascular luminal diameter and area measurements with IVUS has been demonstrated (9-12). Measurements of luminal diameters and areas during the cardiac cycle provide
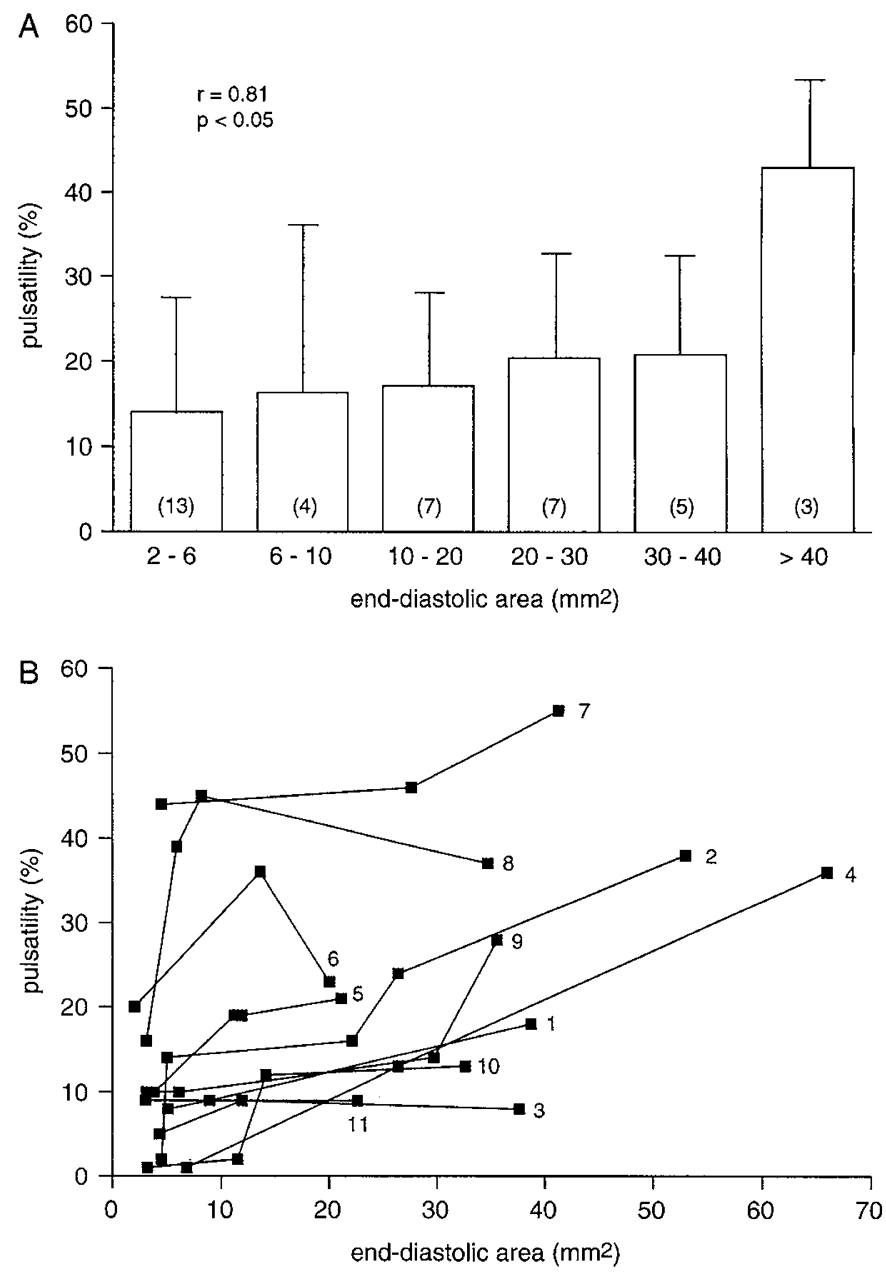

Figure 3. Pulsatility in relation to vessel size in 11 patients. $A$, Measurements of the total patient group, expressed as mean $\pm \mathrm{SD}$. $n=$ number of measurements. $B$, Measurements of the individual patients. $n$, patient number as shown in Table 1.

the possibility of studying pulmonary vascular dynamics in vivo in a unique, direct way.

The pulsatility of the pulmonary artery is one expression of this dynamics. We found a significant relationship between vessel size and pulsatility. Although the pattern of increasing pulsatility toward the proximal arteries was present in all patients, the variation between the individual patients was considerable. One might speculate that the diminished pulsatility in the smaller vessels is caused by a progressive obstruction by the ultrasound catheter in these vessels, thus affecting their responses. However, the interindividual variation, specifically in vessels at similar sites, with similar area in patients with similar weights, makes this hypothesis unlikely. This far more suggests that, as expected, there are more factors than size that affect pulsatility. Pulsatility will be determined by two major components: 1) mechanical forces working on the vessel wall and 2) intrinsic viscomechanical properties of the vessel wall.

Mechanical forces. In vivo, various mechanical forces have their effects on the vascular wall. Radially directed forces, such as pulmonary artery or pulse pressure and the contradirected intrapulmonary pressure, will affect wall dynamics. Also pres- 


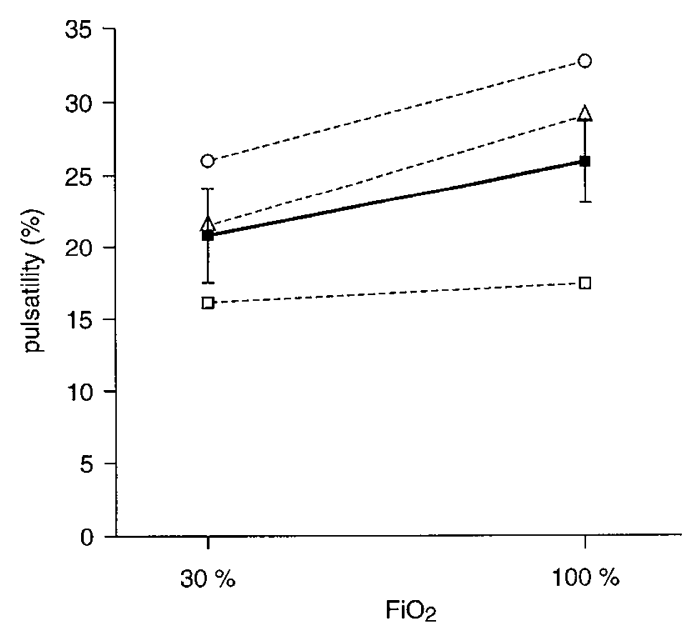

Figure 4. Effect of $100 \%$ oxygen inhalation on pulsatility in six patients. $\mathrm{FiO}_{2}$, percentage of inspired oxygen. Vessel end-diastolic diameter: $1-3 \mathrm{~mm}$, $n=7(\square)$; 3-6 mm, $n=6(\triangle)$; 6-9 mm, $n=3(\bigcirc)$; total group, $n=16(\square)$. $n=$ number of measurements. Pulsatility is expressed as mean \pm SEM for the total group. The increase in mean pulsatility at $100 \%$ inspired oxygen concentration was statistically significant $(p<0.05$, Wilcoxon signed rank sum test for total group).

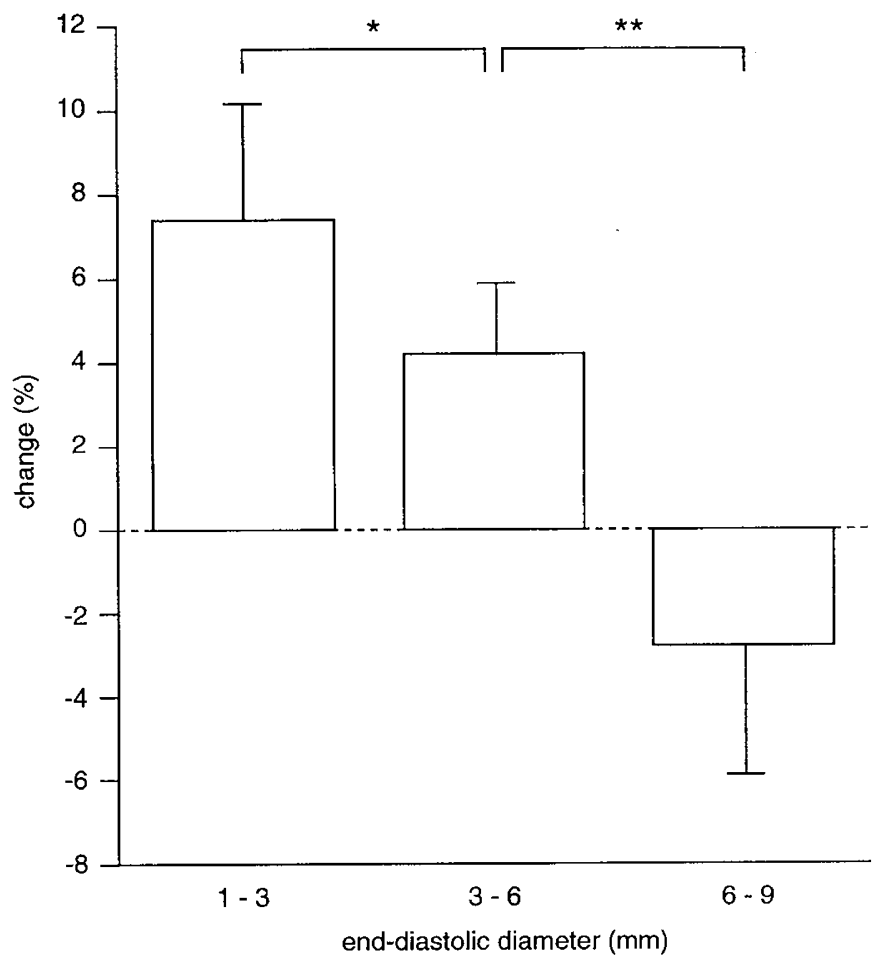

Figure 5. Effect of $100 \%$ oxygen inhalation on end-diastolic vessel diameter. Percentage change in diameter expressed as means \pm SD. Vessels of various diameters showed a statistically significant different effect of $100 \%$ oxygen inhalation $\left(t\right.$ test; $\left.{ }^{*} p<0.05 ;{ }^{* *} p=0.001\right)$.

sure wave reflections, originating from the pulmonary microcirculation and branching of larger vessels, will contribute to the pulsatility, varying with propagation down the pulmonary branches (14). Next, tangential forces, in both longitudinal and circumferential directions, such as shear stress, will affect the mechanics of the vascular wall $(15,16)$. In pulmonary vascular disease these mechanical forces and their effect on wall dynamics will alter. For instance, in the normal pulmonary circulation wave reflections are of small amplitude because of excellent matching of impedances in the distal pulmonary vessels. However, in pulmonary vascular disease these reflection waves will increase in amplitude as well as in velocity with which they return, resulting in an increasing effect on vessel wall dynamics $(14,17)$. It has been demonstrated that mechanical forces have their effects on the metabolic activity in the vascular wall, resulting, on the short term, in adaptation of vascular tone and, on the longer term, changes in the elastic properties of the wall itself $(18-20)$.

Properties of the vessel wall. Referring to the vessel wall itself, this exhibits an anisotropic, nonlinear, viscoelastic mechanical behavior, which varies along the pulmonary branch $(15,16)$. This implicates a very complex mechanical behavior, for which complete description, in fact, over 20 parameters would be needed (15). The dynamic properties of the peripheral, so-called resistance vessels will have a major impact on the pulmonary vascular resistance (17). In the more proximal vessels, often unjustly considered to be conduit arteries with only a passive role in the circulation, these dynamic properties are major determinants not only for the pulsatile load of the right ventricle but also for the shear stress in the arterioles (14, $16,17,21)$. Pulmonary vascular disease affects the dynamical properties of the whole pulmonary vascular tree and therefore will influence the mechanical behavior of peripheral as well as proximal vessels $(19,21)$.

The pulsatility of a vessel is the net result of all described variables and their interactions. Therefore, it might be clear that the interpretation of vessel pulsatility or the prediction of mechanical behavior of blood vessels in general is hazardous. In this study IVUS provided the possibility to assess accurately, in the individual patient, pulmonary artery pulsatility. Thus, it provides unique, direct information on the functional state of the pulmonary arteries, down to a diameter of $1.6 \mathrm{~mm}$. Moreover, the challenge of unraveling the specific contribution to pulsatility of each of the different variables in vivo, will increase the understanding of vascular biophysics.

An additional way of assessing the functional state of the vasculature was to study the effect of a pulmonary vasodilator on vascular dynamics. We found an increase in pulsatility most prominent in the larger vessels. Moreover, with IVUS it was possible to actually visualize vasodilation of the pulmonary vessels directly in vivo. All investigated children showed a vasodilatory reaction, which was the most prominent in the smallest arteries. The slight decrease in diameter after oxygen inhalation, found in the largest arteries, possibly reflects a decrease in diastolic afterload, caused by the dilatation in the smallest vessels. As far as we know, this is the first time vasodilatation has been directly visualized in the pulmonary vasculature in children in vivo. It provides the opportunity to evaluate the response of the pulmonary vessels to different medical interventions in a direct way, avoiding misinterpretations of concomitant events such as pulmonary vessel recruitment. This will have great implications for studies on pulmonary vascular responses in vivo, because currently all investigational methods are using indirect parameters to estimate dilatatory vascular effects or less reliable methods such as angiographic diameter determination $(2,20)$. 
In our experience, the adventitial border could not be delineated well, probably because the pulmonary vessels are embedded in air, frustrating quantitative assessment of the vessel wall thickness. This confirms the limited experience reported in adult patients (22). However, qualitative aspects of the vascular wall could be studied. The inability to distinguish the three layer structure of the wall corresponds with the fact that we studied elastic arteries $(12,23)$. However, a gray inner layer was identified at the inner border of the vessel wall of the one patient with irreversible PVD, which might reflect intima thickening. A congruency may exist with IVUS aspects of coronary intima proliferation in adult patients $(24,25)$. However, histologic validation was not performed in this patient. Few earlier reports claim to distinguish qualitative changes in the vessel wall in pulmonary hypertension $(22,26,27)$. We think larger series, including study of normal pulmonary arteries, are needed to evaluate the value of this qualitative vessel wall assessment.

The smallest vessels we reached with the ultrasound catheter had a diameter of $1.6 \mathrm{~mm}$. In PVD the most striking changes, from a morphologic point of view, occur in the muscular arteries and smaller arterioles, which are vessels with a diameter of less than $1 \mathrm{~mm}$ (23). Although new ultrasound catheters, with a diameter less than $1 \mathrm{~mm}$, are about to become available, the vessels accessible for ultrasound imaging will not be the "resistance vessels." However, as stated before, PVD is a process of the whole pulmonary vasculature, which is not restricted to the small peripheral vessels, but also affects the larger pulmonary arteries $(19,21)$. Every functional change in the pulmonary microcirculation will directly affect the dynamic behavior of the more proximal vessels. The large and medium sized pulmonary arteries are not just passive conduit vessels, but represent an important determinant of integrated pulmonary circulation $(14,17)$. So, their mechanical behavior in PVD is of major interest. In contrast to both hemodynamic and histologic evaluation, IVUS imaging has the possibility to assess vessels of different sizes separately.

In this feasibility study the patient number was too small, with regard to the number of hemodynamic parameters, to perform adequate analysis of possible relationships between hemodynamic variables and ultrasound data. However, there was a tendency of less pulsatile arteries in the patients with elevated pulmonary vascular resistance. Furthermore, in all previously described relations and responses, patient 11, with severe, irreversible PVD, showed aberrant patterns. This suggests the ability of intravascular ultrasound to assess altered vascular dynamic behavior in pulmonary vascular disease.

At the moment we are performing a prospective study in children with congenital heart disease, including not only the various stages of PVD but also normal pulmonary vasculature, in which we will correlate IVUS data with complete hemodynamic, angiographic, and histologic evaluation. This study will demonstrate whether the described advantages of IVUS imaging will have additional value to the currently available diagnostic techniques, in assessing the state of the pulmonary vasculature for clinical as well as research purposes.
We conclude that IVUS imaging of the proximal and peripheral pulmonary arteries is feasible in children and infants using routine catheter techniques. This technique provides a unique opportunity to assess the state of the pulmonary vasculature and its dynamics in vivo directly. Therefore it may be a valuable tool in evaluating the pulmonary vasculature and its changes and responses in normal and pathologic conditions. The additional value with regard to conventional techniques, such as hemodynamic and histologic evaluation remains to be determined. A prospective, comparative study to answer this question is in progress at our department.

Acknowledgments. The authors thank TD Medical b.v., Eindhoven, and Hewlett Packard, Amstelveen, The Netherlands, for their support in providing the equipment used in this study.

\section{REFERENCES}

1. Collins-Nakai RL, Rabinovitch M 1993 Pulmonary vascular obstructive disease. Cardiol Clin 11:675-687

2. Hoffman JIE 1972 Diagnosis and treatment of pulmonary vascular disease. Birth Defects 8:9 -17

3. Haworth SG 1987 Understanding pulmonary vascular disease in young children. [editorial] Int J Cardiol 15:101-103

4. Rich S, Brundage BH 1989 Pulmonary hypertension: a cellular basis for understanding the pathophysiology and treatment. J Am Coll Cardiol 14:545-550

5. Bush A, Busst CM, Haworth SG, Hislop AA, Knight WB, Corrin B, Shinebourne EA 1988 Correlations of lung morphology, pulmonary vascular resistance and outcome in children with congenital heart disease. Br Heart y 59:480-485

6. Frescura C, Thiene G, Giulia Gagliardi M, Mazzuco A, Pellegrino PA, Daliento L Biscaglia S, Carminati M, Galluci V 1991 Is lung biopsy useful for surgical decision making in congenital heart disease? Eur J Cardiothorac Surg 5:118-123

7. Yamaki S, Mohri H, Haneda K, Endo M, Akimoto H 1989 Indications for surgery based on lung biopsy in case of ventricular septal defect and/or patent ductus arteriosus with severe pulmonary hypertension. Chest 96:31-39

8. Haworth SG 1987 Pulmonary vascular disease in ventricular septal defect: Structural and functional correlations in lung biopsies from 85 patients, with outcome of intracardiac repair. J Pathol 152:157-168

9. Pandian NG, Hsu TL 1992 Intravascular ultrasound and intracardiac echocardiography: Concepts for the future. Am J Cardiol $69: 6 \mathrm{H}-17 \mathrm{H}$

10. Pandian NG, Weintraub A, Kreis A, Schwartz SL, Konstam MA, Salem DN 1990 Intracardiac, intravascular, two-dimensional, high-frequency ultrasound imaging of pulmonary artery and its branches in humans and animals. Circulation 81:2007-2012

11. Nishimura RA, Edwards WD, Warnes CA, Reeder GS, Holmes DR, Tajik AJ, Yock PG 1990 Intravascular ultrasound imaging: In vitro validation and pathologic correlation. J Am Coll Cardiol 16:145-154

12. Gussenhoven EJ, Essed CE, Lancee CT, Mastik F, Frietman P, van Egmond F, Reiber J, Bosch H, van Urk H, Roelandt JRTC, Bom N 1989 Arterial wall characteristics determined by intravascular ultrasound imaging: An in vitro study. J Am Coll Cardiol 14:947-952

13. Rabinovitch M, Keane JF, Fellows KE, Castaneda AR, Reid L 1981 Quantitative analysis of the pulmonary wedge angiogram in congenital heart defects. Circulation 63:152-164

14. Sniderman AD, Fitchett DH 1988 Vasodilators and pulmonary hypertension: the paradox of therapeutic success and clinical failure. [editorial] Int J Cardiol 20:173181

15. Lee RT, Kamm RD 1994 Vascular mechanics for the cardiologist. J Am Coll Cardiol 23:1289-1295

16. Winlove CP, Parker KH 1993 Vascular biophysics: Mechanics and permeability. Eur Respir Rev 3:535-542

17. Ramsey MW, Jones CJH 1994 Large arteries are more than passive conduits. [editorial] Br Heart J 72:3-4

18. Gibbons GH, Dzau VJ 1994 The emerging concept of vascular remodeling. N Engl J Med 330:1431-1438

19. Riley DJ, Poiani GJ, Wilson FJ, Thakker-Varia S, Tozzi CA 1993 In vivo and in vitro evidence for the involvement of mechanical forces in pulmonary artery tissue remodeling. Eur Respir Rev 3:609-612

20. Celermajer DS, Cullen S, Deanfield JE 1993 Impairment of endothelium-dependent pulmonary artery relaxation in children with congenital heart disease and abnormal pulmonary hemodynamics. Circulation 87:440-446

21. Reeves JT, Durmowicz AG, Weiser MCM, Orton EC, Stenmark KR 1993 Diversity in the pulmonary circulation: An overview of the international conference on the pulmonary vasculature in health and disease. Eur Respir Rev 3:530-534

22. Porter TR, Taylor DO, Fields J, Cycan A, Akosah K, Mohanty PK, Pandian NG 1993 Direct in vivo evaluation of pulmonary arterial pathology in chronic congestive 
heart failure with catheter-based intravascular ultrasound imaging. Am J Cardiol 71:754-757

23. Wagenvoort C.A. Mooi WJ 1989 Biopsy Pathology of the Pulmonary Vasculature Chapman \& Hall, London

24. Di Mario C, The SHK, Madretsma S, van Suylen RJ, Wilson R, Bom N, Serruys PW, Gussenhoven WG, Roelandt JRTC 1992 Detection and characterization of vascular lesions by intravascular ultrasound: An in-vivo correlative study with histology. J Am Soc Echocardiogr 19:135-146
25. Porter TR, Sears T, Xie F, Michels A, Mata J, Welsh D, Shurmur S 1993 Intravascular ultrasound study of angiographically mildly diseased coronary arteries. J Am Coll Cardiol 22:1858--1865

26. Scott PJ, Essop AR, Al-Ashab W, Deaner A, Parsons J, Williams G 1993 Imaging of pulmonary vascular disease by intravascular ultrasound. Int J Card Imaging 9:179184

27. Kravitz KD, Scharf GR, Chandrasekaran K 1994 In vivo diagnosis of pulmonary atherosclerosis. Role of intravascular ultrasound. Chest 106:632-634 